\title{
Protocol for Systematic Review on Inequity in Child Health Care Service Utilization in Low and Middle- income Countries
}

Hailu Fekadu Demessie ( $\square$ hailufekadu18@yahoo.com )

Arsi University College of Health Scince Department of Public Health

Damen Hailemariam Hailemariam

Addis Ababa University

Helmut Kloos Kloos

Californea

Aynalem Adugna Adugna

California

Wubegzier Mekonnen Mekonnen

Addis Ababa University

\section{Protocol}

Keywords: Child health care, Health services utilization, LMICs,Inequities, Systematic review, Protocol

Posted Date: October 28th, 2020

DOl: https://doi.org/10.21203/rs.3.rs-96737/v1

License: (9) This work is licensed under a Creative Commons Attribution 4.0 International License. Read Full License 


\section{Abstract}

Background: One challenge to achieving Millennium Development Goals was inequitable access to quality health services. In order to achieve the Sustainable Development Goals, interventions need to reach underserved populations, it appears that the maternal, newborn and child health goals (MDG 4 and 5) will not be universally achieved. There was early recognition that it could be possible to achieve the health goals while decreasing health inequity, because most of the gains might go to the better-off rather than to the very poor.

Methodology/Design: The current protocol adopts a strategy informed by the guidelines of The Cochrane Handbook for Systematic Reviews. Our systematic review will identify studies in English, provided inclusion of an English abstract - from 2010 onwards till 2020, by searching MEDLINE (PubMed interface), EMBASE (OVID interface), Cochrane Central (OVID interface) and the gray literature. Study selection criteria include research setting, study design, reported outcomes and determinants of interest. Our primary outcome is inequity in utilization of child health care services, and determinants of concern are: 1) socioeconomic status (for example, income, education); 2) geographic determinants (for example, distance to a health center, rural versus urban residence); and 3) demographic determinants (for example, age, ethnicity, religion, and marital status). Screening, data abstraction, and scientific quality assessment will be conducted independently by two reviewers using standardized forms. Where feasible, study results will be combined through meta-analyses to obtain a pooled measure of association between utilization of child health care services and key determinants. Results will be stratified by income levels (World Bank classification) geographical residence and demographical determinants.

Discussion: Our review will inform policy-making with the aim of decreasing inequities in utilization of child health care services. This research will provide evidence on unmet needs for child health care services in LMICs, knowledge gaps and recommendations to health policy planners. Our research will help promote universal coverage of quality child health care services as an integral part of the continuum of maternal and child health care. This protocol will be registered with the Prospero database.

\section{Background}

In 2012 it was estimated that 6.6 million children under the age of five died worldwide [1], eighty-two per cent of which in Sub-Saharan Africa and Southern Asia. Thirty-three per cent of these deaths were due to pneumonia (17\%), diarrhoea (9\%) and malaria (7\%) (2).

Progress in both intervention and impact indicators of child health have been inconsistent over several decades, with several evidences showing both between and within country disparities in several of these child health indicators [1, 2]. These disparities are especially significant in Low and Middle-Income Countries (LMICs), with 99\% of global child and neonatal death occurring in these countries [3].

Evidence also shows that poor health outcomes at national levels are highly reflective of disparities at individual household levels; such reflection was demonstrated in the work of Chao and colleagues [4, 5], 
exploring relationships between household wealth quintiles and Under-Five Mortality Rates (U5MR). Access to important health care services, as well as the overall health outcome of women and children, in LMICs often depends on factors such as gender of a child,

place of residence, level of education, income and other socio-economic parameters $[1,5]$. These factors collectively referred to as 'dimensions of inequality'; usually have an interwoven effect. Low income level for example, may directly prevent a woman from utilizing health services due to associated medical, nonmedical and opportunity costs $[3,5]$, equally a low level of educational attainment may also imply that there is limited economic opportunities, hence impaired ability to earn sufficient income. Low socioeconomic status may also result in women and children living in areas with limited health infrastructure [6].

To effectively assess coverage, and progress in child health, changes in inequity must be monitored over time[7], several indexes and frameworks have been developed for this purpose [7]. These frameworks often comprise of important child health services and outcome indicators. Examples of these include the composite coverage index [8, 9], co-coverage index, count down to 2015 indicator framework [10].

Despite the substantial body of literature that have explored equity dimensions for the various child health indicators $[11,12]$, there remains a significant gap in literature, which concerns the paucity of systematic reviews and meta-analysis synthesizing evidence from the various existing cross-sectional studies. Furthermore, the lack of household survey data for certain interventions implies there are no equity studies for those interventions. For example, no study has investigated inequality in the availability of ARV prophylaxis for pregnant women with HIV/AIDS to prevent HIV transmission to the new born, this may be explained by the fact that information is not available in any database that allows equity breakdown for this indicator [2]. Likewise, there is a paucity of study on the effect of inequality on existing studies only examined inequity in child health service at national levels, and not from an individual or household level, for example there are studies examining variation in maternal and child mortality based on national Gross National Income (GNI) or Gross Domestic product (GDP) levels [12], or based on Human Development Index (HDI) [2, 12], no study has examined child health care service utilization based on individual household wealth index. This lack of household-level data disaggregation, is also likely due to the fact that child mortality is currently estimated through modelling, at national levels only, and equity breakdowns are not available [2].

The objective of our study is to fill the gap concerning the lack of systematic review and meta-analysis, and to provide a pooled evidence base on the association between a selection of child health indicators, and three important equity stratifies, with focus on studies that utilized nationally representative data. Other gaps identified regarding the paucity of studies on child health

Objectives and research question

Our Objectives are to: 
1. Systematically identify and asses' studies and reports on the utilization of child health services.

2. Synthetize evidence on the determinant of child health services utilization and inequity in the use of child health services

3. Provide evidence to policy planners in order to address unmet need for child health services utilization.

This systematic review is guided by the following research questions.

Is child health service utilization associated with 1 . Socioeconomic 2. geographic and 3. demographic determinants?

\section{Methodology/design}

The current protocol outlines a strategy informed by the guideline of the Cochrane collaboration (Cochrane hand book of for systematic review) [13]. The systematic review will follow the four phases flow diagram (Fig. 1) put forth by the preferred reporting items for systematic reviews and meta-analysis (PRISMA) statement [14].

\section{Information sources and literature search}

Literature search strategies will be implemented by the search team of Addis Ababa University

Filters for bibliographic research will include publication date - from 2010 onwards till 202 and published in English language. We will use specific medical terms/ subjects headings (MeSH) term and text words to identify study by searching MEDLINE (Pubmed interface).

We will hand -search relevant abstracts in the Cochrane child group ,Cochrane pregnancy, child birth and public health group. As per peer review of electronic of Electronic search strategies (PRESSS) recommendation, we will include the 'explode' option to the entree terms in the EMBASE research. The exact search strategy for EMBASE, MEDLINE and Cochrane central can be found. We will search the gray literature namely the following sources WHO, World Bank and UNICEF.

We utilized a framework of child health care indicators from the 2010-2015 global strategy for women's and children's health [12], comprising of two mortality indicators, eight coverage indicators and one child nutrition indicators as follows: comprising of eight measures of intervention coverage, and three measures of impact as follows: U5MR, stunting in children under five, exclusive breastfeeding, three doses of combined diphtheria-tetanus-pertussis immunization coverage (DPT3), and care seeking for suspected pneumonia, fever and diarrhea [13].

We categorized the included indicators into two broad groups; negative indicators, and positive indicators. The negative indicators were prevalent among the poor, these includes: U5MR, stunting in children under five, while the positive indicators are mostly pro-rich, which includes: exclusive breastfeeding, three doses of combined diphtheria-tetanus- pertussis immunization coverage (DPT3), and 
care seeking for suspected pneumonia and diarrhea [13]. The review protocol will be registered at the PROSPERO international prospective register of systematic reviews.

\section{Ethics}

This study analyzed secondary data and exempted from ethics review by the Griffith University office for research.

Search strategy

The online databases PubMed, Embase, Scopus will be searched form 2010 until June 2020 for relevant studies, we also search the publications of the World Bank, WHO, and UNICEF. The following key words will be used for the search: Gaps OR Disparity AND Child Mortality AND Developing Countries, Inequity OR Inequality AND Child Mortality AND Developing Countries, Gaps OR Disparity AND Under-five Mortality AND Developing countries, Inequality OR Inequity AND Under-five Mortality AND Developing Countries, Gaps OR Disparity AND Stunting in children AND Developing countries, Inequality OR Inequity AND Stunting in children AND Developing countries, Gaps OR Disparity AND Exclusive breastfeeding AND Developing countries, Inequality OR Inequity AND Exclusive Breastfeeding AND Developing countries, Gaps OR Disparity AND DPT3 immunization AND Developing countries, Inequality OR Inequity AND DPT3 immunization AND Developing countries Gaps OR Disparity AND Care seeking for children with suspected pneumonia AND Developing countries, Inequality OR Inequity AND Care seeking for children with suspected Pneumonia And Developing countries. The Preferred Reporting Items for Systematic Reviews and Meta- Analysis guidelines will be followed in searching the results.

Inclusion criteria

Participants and settings

We will retrieve studies implemented in LMICs, as defined by The World Bank Group's classification [15]. We will extracted data from studies that met the following inclusion criteria: 1) Publication in peerreviewed literature in the last 10 years (2010-2020); 2) Study must be based on data from at least one nationally representative data; 3 ) Study must have a clearly defined primary outcome, encompassing one or more of the eleven pre-defined core child health indicator; 4) Study must have investigated the effect of at least one of three 'dimensions of inequity', which includes: Income level, level of education and place of residence; 5) Data from low or middle-income countries only must have been utilized; 6) Included studies must have utilized a quantitative analytical method

\section{Exclusion criteria}

We will exclude studies based on the following criteria: 1) Studies that did not use a nationally representative data; 2) Studies that were not peer-reviewed; 3) Studies that did not assess at least one of the 3 dimensions of inequity: place of residence, income level and level of education; 4) Studies that did 
not include at least one of the child health outcome measures; 5) Studies that used data from high income countries; 6) Studies that were purely descriptive.

\section{Design}

Our systematic review will include all observational studies including cohort, case-control and crosssectional studies

\section{Determinants}

Determinants of concern are: 1) socioeconomic status -assessed by income, expenditure, household characteristics and/or assets, occupational or contractual status and education (highest level of education completed, years of schooling, literacy 2) geographic determinants (Euclidian distance - km - to a health center, travel time, location - rural versus urban residence) and 3) demographic determinants ethnicity, marital status, age.

\section{Results}

We will consider quantitative results of the association between potential determinants and the utilization of child health care services. Published results must include an association measure, frequency ratio/difference, or statistical test comparing utilization of child health care services across two or more groups.

If these results are not explicit, we have to be able to estimate them with the information provided in the paper. We will consider relative comparisons - for example, relative concentration index $(\mathrm{RCl})$ or relative index of inequity (RII) - to a reference group, along with absolute differences in child health care services utilization, such as absolute concentration index (ACI) or slope index of inequity (SII). Such reported disparities will be useful in making comparisons over time or across geographical areas, populations or indicators, in light of the Centers for Disease Control and Prevention (CDC)'s guidelines [16, 17]. Studies strictly reporting qualitative results on access to child health care utilization are excluded. Within the same publication, results for the most recent year will be appraised if information exists for consecutive years. In the case of secondary analyses from national representative surveys such as the Demographic and Health Surveys (DHS) for consecutive years in the same country, we will only consider the most recent .

\section{Study selection procedure}

\section{Screening}

A team of researchers, HF will identify articles by first analyzing titles and abstracts for relevance and compliance with the selection criteria, based on research setting, study design, reported outcomes and 
determinants of interest. Relevant articles will be classified as: 1) included; 2) excluded; or 3) uncertain. After exclusion of records not relevant to the systematic review, full texts of selected abstracts (records categorized as included or uncertain) will be extracted systematically for further eligibility analysis.

\section{Eligibility}

Full-text screening will be conducted independently by the reviewers (HF) using a standardized form with explicit inclusion and exclusion criteria. Discrepancies will be resolved by discussion between the two reviewers, and persisting disagreement will be resolved by discussions with two experienced researchers.

\section{Data collection process}

Reviewers will use an explicit data collection form to abstract data items, including but not limited to: study characteristics (country, setting, year of publication, study design, sample size); participants' characteristics (mean age $\pm S D$, health literacy, women's decision making power); outcomes (child health care service utilization); and results of the association between child health care services and potential determinants.

In cases where numerous publications report data originating from the same study, the latest outcomes of interest will be assessed. Missing data on key characteristics will be dealt with by contacting the study authors and through complementary research. Reviewers will systematically use a standardized data abstraction form. To increase the reliability of data abstraction by the reviewers, a pilot test of the standardized form will be performed on a random sample, and the tool will be refined as necessary.

Methodological quality assessment

We will assess the scientific quality of selected studies to ensure internal validity of reported results and avoid analyzing false associations - confounded or biased - or type I statistical errors. We will use standardized quality assessment tools for specific types of designs to determine the methodological quality and the risk of bias of the included studies. To assess the quality of cohort, case control and cross-sectional studies, we will use the Effective Public Health Practice Project (EPHPP) Quality Assessment Tool for Quantitative Studies, adapted to extend the criteria for selection bias assessment [18].Special attention will be provided to precise study objectives, explicit identification of the population studied, clear definitions of outcomes, independent factors, potential confounders and effect modifiers. According to the methodological characteristics appraised, we will classify the studies' scientific quality as either 1) Strong, 2) Moderate or 3) Weak.

\section{Search results}

Evidence tables will be generated to descriptively summarize the included studies and results: 1) authors, 2) study design, 3) objectives, 4) setting, 5) population, 6) outcomes assessed, 7) determinants/predictors, 8) results and 9) scientific quality. Evidence tables will be stratified 
by countries' income level (World Bank classification) to provide for different contextual characteristics of low versus middle-income countries.

Data synthesis

Where feasible, data will be combined to obtain a pooled measure of association evaluating child health care services inequities, through meta-analyses conducted by' using The Cochrane Group's Review Manager Software (RevMan 5.1) [19]. Data will be analyzed along subsets defined by the countries' income level and grouped by determinants of child health care services utilization (socioeconomic, geographic, demographic). Due consideration will be given to heterogeneity (12 statistic) and corresponding analysis (fixed versus random-effects models; meta-regression, if necessary). Depending on the number of studies, we will further stratify observational studies according to design (cohort, casecontrol, cross-sectional) and/or association measure - odds ratio, risk ratio, incidence rate ratio, hazard ratio, and prevalence ratio - exploring potential heterogeneity.

will be paid to assessing results in light of study settings to ensure proper contextualization of evidence and relevance for policy planning purposes in LMICs. Results will be reported according to the PRISMA Statement, with a focus on health equity (PRISMA-Equity 2012 Extension) [20].

\section{Discussion}

This systematic review will provide: 1) knowledge on existing inequities and unmet needs for child health care services in LMICs; 2) pragmatic recommendations to health policy planners for improving access to, and utilization of, quality child health care service in LMICs; and 3) an overview of knowledge gaps and future research needs. Results of the systematic review will be published in a peer-reviewed international journal and presented at conferences and symposia in relevant fields (for example, global health, health policy and planning, health systems, healthcare equity). Further knowledge dissemination will involve communicating results to the governments of LMICs and to organizations active in promoting access to maternal and child health services (for example, WHO, Family Care International).

The utmost relevance of systematic reviews to inform health systems policymaking is increasingly recognized. Tugwell et al. (2010) underlined that a focus on health equity in systematic reviews improves their relevance for public policy making [18]. Welch et al. (2012) stressed that systematic reviews are a valuable source of scientific evidence on inequities in health outcomes, resource allocation and use [20]. Our review will hence supply evidence to health policy planners with the objective of decreasing inequalities in maternal and child health indicators and promoting universal coverage of essential obstetric care services. Knowledge thus created may help promote equitable access to child health care services as a fundamental element of the continuum of care essential to reduce child mortality and morbidity.

\section{List Of Abbreviations}


CDC-Center for disease control and prevention

DHS- Demographic health survey

DPT3- Diphtheria, Pertussis and Tetanus

EPHPP -Effective Public Health Practice Project

GDP - Gross Domestic product

GNI -Gross National Income

HDI -Human Development Index

HIV/AIDs- Human Immunodeficiency syndrome

LMICs-Low and middle income countries

MDG- Millennium developmental goals

$\mathrm{RCl}$ - Relative concentration index

UNICEF - United Nation international fund for children

WHO- World health Organization

\section{Declarations}

\section{Ethics approval and consent to participate}

This study analyzed secondary data and exempted from ethics review by the Griffith University office for research

\section{Consent for publication}

\section{Availability of data and materials}

Data and materials are available and will be submitted per request

\section{Competing interests}

The Authors confirm they have no competing interest

\section{Funding}

No funding agency for this review 


\section{Authors' contributions}

$\mathrm{HF}, \mathrm{DH}, \mathrm{WM}$ Design, conceptualize the protocol, data extraction, analyze and wrote the report HK and AA analyze and edit the whole document, all researchers read and approve the protocol for publication

\section{Acknowledgements}

We acknowledged all individuals who had given us training and knowledge on systematic review especially Addis Ababa University Staffs

\section{References}

1. Victora CG, Requejo JH, Barros AJD, Berman P, Bhutta Z, Boerma T, et al. Countdown to 2015: a decade of tracking progress for maternal, newborn, and child survival. Lancet. 2016;387:2049-59. Medline:26477328 doi:10.1016/S0140-6736(15)00519-X

2. Keats EC, Ngugi A, Macharia W, Akseer N, Khaemba EN, Bhatti Z, et al. Progress and priorities for reproductive, maternal, newborn, and child health in Kenya: a Countdown to 2015 country case study. Lancet Glob Health. 2017;5:e782- 95. Medline:28716350 doi:10.1016/S2214-109X(17)30246-2

3. Trends in maternal mortality:1990 to 2013. Estimates by WHO, UNICEF, UNFPA, The World Bank and United Nations Population Division. Geneva; 2014.

4. Tajik P, Nedjat S, Afshar NE, Changizi N, Yazdizadeh B, Azemikhah A, et al. Inequality in maternal mortality in iran: an ecologic study. Int J Prev Med. 2012;3:116-21. Medline:22347608

5. Gabrysch SCO. Still too far to walk: Literature review of the determinants of delivery service use. BMC Pregnancy Childbirth. 2009;9:34. Medline:19671156 doi:10.1186/1471-2393-9-34

6. World Health Organization. State of Inequality: Reproductive Maternal Newborn and Child Health: Interactive Visualization of Health Data. Geneva: WHO; 2015.

7. Boerma JT, Bryce J, Kinfu Y, Axelson H, Victora CG, Victora CG; Countdown 2008 Equity Analysis Group. Mind the gap: equity and trends in coverage of maternal, newborn, and child health services in 54 Countdown countries. Lancet. 2008;371:1259-67.Medline:18406860 doi:10.1016/S01406736(08)60560-7

8. Barros AJ, Victora CG. Measuring coverage in $\mathrm{MNCH}$ : determining and interpreting inequalities in coverage of maternal, newborn, and child health interventions. PLoS Med. 2013;10:e1001390.Medline:23667332 doi:10.1371/journal.pmed.1001390

9. Victora CG, Fenn B, Bryce J, Kirkwood BR. Co-coverage of preventive interventions and implications for child-survival strategies: evidence from national surveys. Lancet. 2005;366:14606.Medline:16243091 doi:10.1016/S0140-6736(05)67599-X

10. Countdown to 2015. Tracking progress in child survival: the 2005 report. New York, NY: UNICEF; 2005. 
11. Afnan-Holmes H, Magoma M, John T, Levira F, Msemo G, Armstrong CE, et al. Tanzania's Countdown to 2015: an analysis of two decades of progress and gaps for reproductive, maternal, newborn, and child health, to inform priorities for post-2015.

12. Adegbosin A, Sun J, Stantic B, Sen Wang HZ. Systematic review and meta-analysis of the effects of inequality on the core indicators of reproductive, maternal, newborn and child health. PROSPERO 2018.

13. Cochrane Collaborative: InCochrane Handbook for Systematic Reviews of Interventions, version 5.1.0 [Updated March 2011]. Edited by Higgins JPT, Green S; 2011. Available from: http://www.cochranehandbook.org

14. Moher D, Liberati A, Tetzlaff J, Altman DG, PRISMA Group: Preferred reporting items for systematic reviews and meta-analyses: the PRISMA statement. BMJ 2009,339:b2535

15. The World Bank: Country and Lending Groups; 2013. [cited 25 June 2013]; Available from: http://data.worldbank.org/about/country-classifications/ country-and-lending-groups.

16. Keppel K, Pamuk E, Lynch J, Carter-Pokras O, Kim I, Mays V, Pearcy J, Schoenbach V, Weissman JS:Methodological issues in measuring health disparities. Vital Health Stat2005,2(141):1-16.

17. Tugwell P, Petticrew M, Kristjansson E, Welch V, Ueffing E, Waters E, Bonnefoy J, Morgan A, Doohan E, Kelly MP:Assessing equity in systematic reviews: realising the recommendations of the commission on social determinants of health.BMJ2010,341:c4739.

18. Effective Public Health Practice Project:Quality Assessment Tool for Quantitative Studies; 2013. Available from: http://www.ephpp.ca/PDF/Quality \%20Assessment\%20Tool_2010_2.pdf

19. Cochrane Information Management System (IMS):Review Manager (RevMan) version 5.1; 2011. Available from: http://ims.cochrane.org/revman.

20. Welch V, Petticrew M, Tugwell P, Moher D, O'Neill J, Waters E, White H, PRISMA-Equity Bellagio group:PRISMA-Equity 2012 extension: reporting guidelines for systematic reviews with a focus on health equity. PLoS Med2012,9:e1001333.

\section{Figures}




\section{Identification}

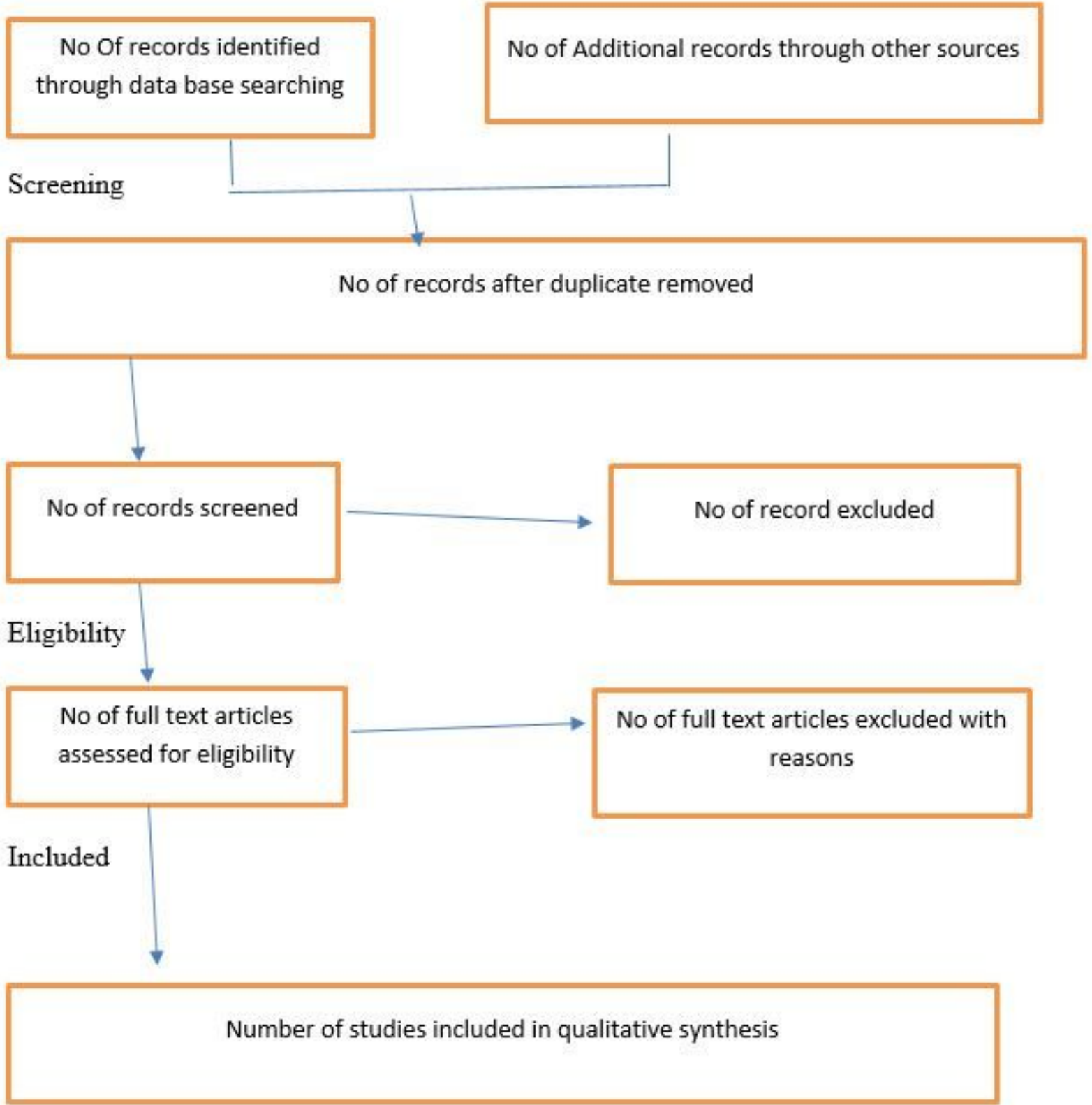

Figure 1

PRISMA Flow Diagram

\section{Supplementary Files}

This is a list of supplementary files associated with this preprint. Click to download.

- PRISMAPchecklist.docx 